\title{
CURRENT SITUATION OF DESIGN OF WALKING MECHANISM CONFIGURATION OF TRACKED MOBILE ROBOT
}

\author{
Liu Jie ${ }^{1}$, Dong Jia Lin ${ }^{2}$, Ding Zi Hao ${ }^{3}$, Fang Zheng Da ${ }^{4}$, Huang Li Xin ${ }^{5}$ \\ ${ }^{I}$ School of Mechanical Engineering, Shanghai University of Engineering Science, Shanghai, China \\ ${ }^{2}$ School of Mechanical Engineering, Shanghai University of Engineering Science, Shanghai, China \\ ${ }^{3}$ School of Mechanical Engineering, Shanghai University of Engineering Science, Shanghai, China \\ ${ }^{4}$ School of Mechanical Engineering, Shanghai University of Engineering Science, Shanghai, China \\ ${ }^{5}$ Engineering Training Center, Shanghai University of Engineering Science, Shanghai, China
}

\begin{abstract}
For the motion characteristics of the tracked mobile robot under the complicated terrain environment, the current design on configuration of walking mechanism for tracked mobile robot are systematically summarized. The comparisons between the fixed tracked and the reconfigurable tracked mobile robot about the typical design on configuration of walking mechanism, the obstacle overcoming ability and characteristics about various configurations of walking mechanism for tracked mobile robot are analyzed and based on method of extrapolated configuration the regulation and connection among various tracked robot are proposed. Finally, the problems of tracked mobile robot and the development trends of the design on configuration of walking mechanism are put forward.
\end{abstract}

Keywords: Tracked Mobile Robot; Topographic Environment; Travelling Mechanism; Reconfigurable; The ObstacleSurmounting Performance.

\section{INTRODUCTION}

Robot technology is gradually turning to the research of special robot technology that can perform tasks in special environments. Mobile robot is the earliest and most widely used special robot.

Robots are a common part of the wheel, crawler, legs, feet and so on to promote car body on the ground in the mobile device, configure these wheel, crawler foot or leg to make it play their function called mobile system course design, can also be called a mobile robot walking mechanism [3-6] design, the design of the robot mechanism configuration is the focus of the mobile robot manipulator design.

As far as the ground mobile robot is concerned, it is generally divided into wheel type, crawler type, leg type, self-reconfiguration type and compound type. Comprehensive comparison, although crawler than wheeled mobile robot structure movement flexibility is low, but the terrain adaptability than wheeled, cross-country performance slightly behind the leg type, the structure than leg type, since the refactoring type, compound control is convenient, simple and has a good grounding than the small, cross-country motor performance, and the advantages of small steering radius, so the crawler type mobile robot has good application prospect, is becoming a hot spot of research.
The configuration design of mobile robot walking mechanism is the foundation of robot research. This paper focuses on the research of walking mechanism configuration of crawler mobile robot. By analyzing the research status of crawler mobile robots at home and abroad, this study makes a comprehensive analysis of various configurations and research defects of robot walking mechanism, and forecasts the development trend of crawler mobile robots.

\section{CURRENT STATUS OF CATERPILLAR STRUCTURE DESIGN}

Based on the comprehensive analysis of the research on the design of crawler mobile robot configuration at home and abroad, the walking mechanism can be divided into two types: single two-track, double four-track, multi-track and multi-track. It can be divided into fixed track type and deformable track type.

\subsection{Design Status of Fixed Crawler Walking Mechanism Configuration}

The fixed crawler type mobile robot is a crawler type robot which keeps its inherent crawler configuration. There are many types of fixed crawler mobile robots with diverse configurations. Typical fixed crawler mobile robots at home and abroad include snow leopard 20 explosive discharge robot, Packbot [8] series developed by American iROBOT company and mobile robot for earthquake rescue. 
Snow leopard 20 explosive discharging robot (as shown in Fig.1), this type of robot is of single joint and double track structure, with simple structure and good motion stability compared with the same type of wheeled type. However, this type of robot is slow in running and has limited off-road performance.

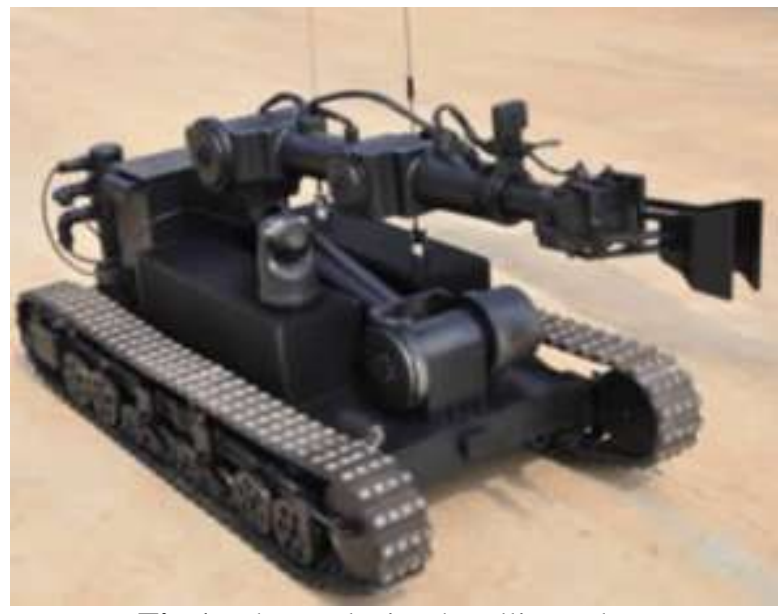

Fig 1: The explosive-handling robot

Packbot (as shown in Fig.2) is a double-jointed four-track structure, with a pair of swing arm crawler in front, which can play an auxiliary role on the main crawler. Compared with single-joint two-track structure, Packbot has good offroad performance, but poor maneuverability compared with the same type of wheeled type.

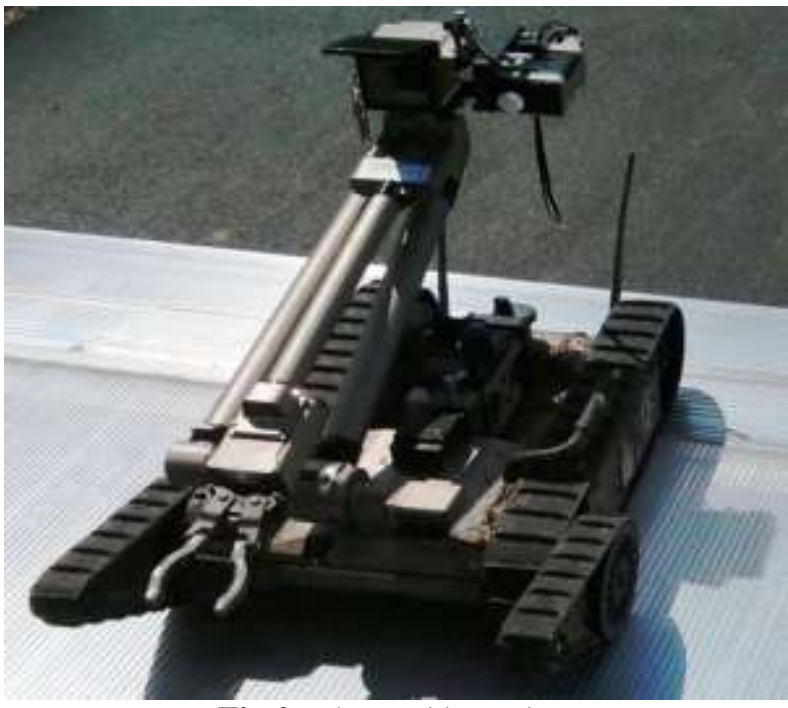

Fig 2: The Packbot robot

The mobile robot for earthquake rescue (as shown in Fig.3) is mainly composed of a mobile unit and an open device. Before and after the moving unit respectively adopt a pair of swing arms to assist the main track to perform various tasks. Its opening device is similar to a hydraulic jack, and the lifting height is $3 \mathrm{~cm} \sim 20 \mathrm{~cm}$ under the load of $1000 \mathrm{~kg}$. The external size of the robot after the opening device is removed is $820 \mathrm{~mm} * 560 \mathrm{~mm} * 260 \mathrm{~mm}$, weighs $40 \mathrm{~kg}$, the maximum driving speed is $0.5 \mathrm{~m} / \mathrm{s}$, and the maximum load is
$50 \mathrm{~kg}$. This type of robot adopts the three-section six-track mechanism, which belongs to the multi-section multi-track structure. Compared with the two-section four-track structure, it has added a pair of swing arms, and its obstacle surmounting performance has been significantly improved.

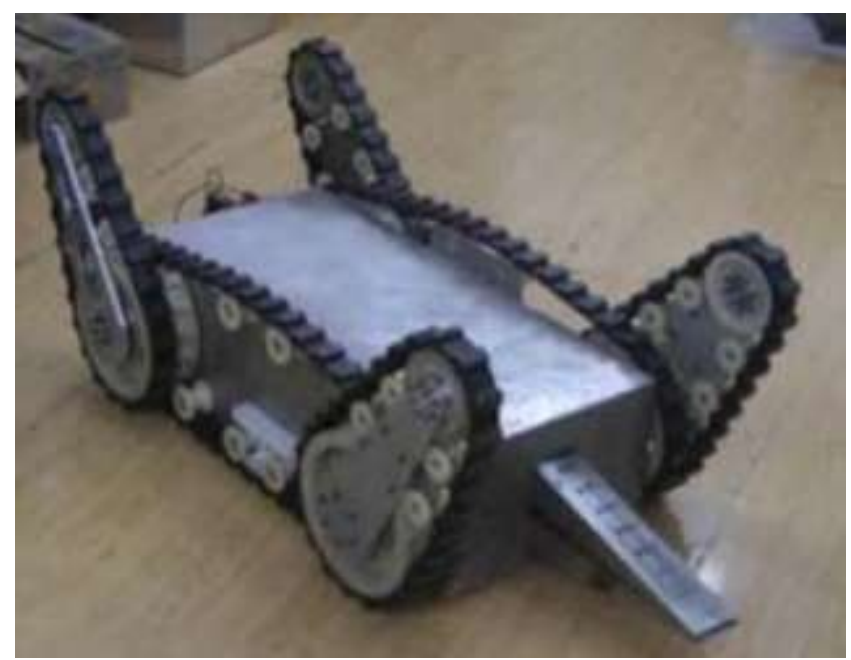

Fig 3: The earthquake rescue mobile robot

In addition to the above structures, fixed crawler mobile robots are also used in some compound and selfreconstruction mobile robots, such as the wheeled compound robot developed by xi 'an university of science and technology and the coal mine search robot designed by the robotics institute of Harbin Institute of Technology.

\subsubsection{Current Status of Design of Flexible Crawler}

\section{Structure}

The so-called deformable crawler mobile robot refers to that the configuration of the crawler used by the robot can be appropriately changed according to topographic conditions and operation requirements. Typical deformable crawler mobile robots at home and abroad, such as RTMBot developed by national university of defense technology, neza-i robot developed by national key laboratory of robotics of shenyang institute of automation, Chinese academy of sciences, and terrain adaptive robot, etc.

RTMBot (as shown in Fig.4) belongs to a single two-track mobile robot, which is composed of a cradle head and a pair of deformable crawlers arranged symmetrically. Each deformable crawler is composed of a crawler, four crawler wheels and deformation device. The crawler wheels are fixed on the connecting rod shaft. The deformable crawler adopts the configuration of four-bar linkage. There are two typical obstacle crossing modes: one is to raise the crawler to cross the obstacle directly by changing the swinging Angle of the connecting rod; the other is to use climbing mode to cross the obstacle without lifting the crawler. The robot's overall size is $700 \mathrm{~mm} \times 500 \mathrm{~mm} \times 300 \mathrm{~mm}$, with maximum climbing Angle of 45 degrees and maximum obstacle climbing height of $200 \mathrm{~mm}$. Compared with the 
same type of fixed crawler robot, the performance of the obstacle surmounting is higher, the deformation of the crawler makes it more flexible, and the ground adaptability is obviously enhanced. However, due to the larger impact when the robot moves at high speed, the performance of the deformable linkage mechanism is required to be higher, and its motion stability is relatively poor compared with the fixed structure.

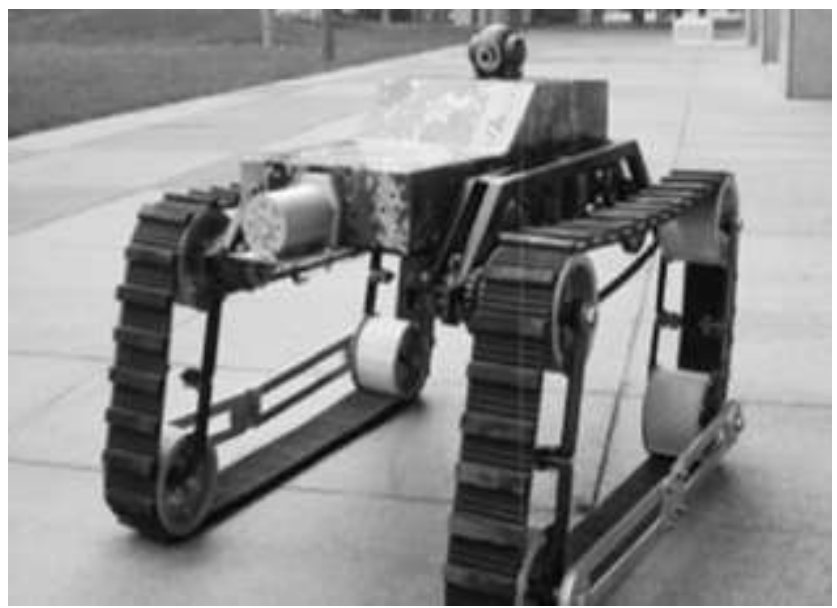

Fig 4: The RTMBot robot

The neza-i robot (as shown in Fig.5) is a compound rotarod mobile robot, and has the ability to deform the crawler. It can realize the change of wheel mode and track mode by adjusting the motion posture according to the different environment. The maximum geometric size of the robot is $289 \mathrm{~mm} \times 360 \mathrm{~mm} \times 146 \mathrm{~mm}$, which can overcome obstacles of $120 \mathrm{~mm}$ height. Compared with the crawler mobile robot with fixed geometric shapes, this robot adopts the driving mode of passive adaptation to the environment. It has good off-road performance, strong ability to overcome obstacles, strong adaptability to non-structural environment and can be widely used. However, the transmission system is arranged within the width range of the crawler, which makes its structure more complex, which has a great impact on the crawler when walking, and requires higher performance of the crawler material.

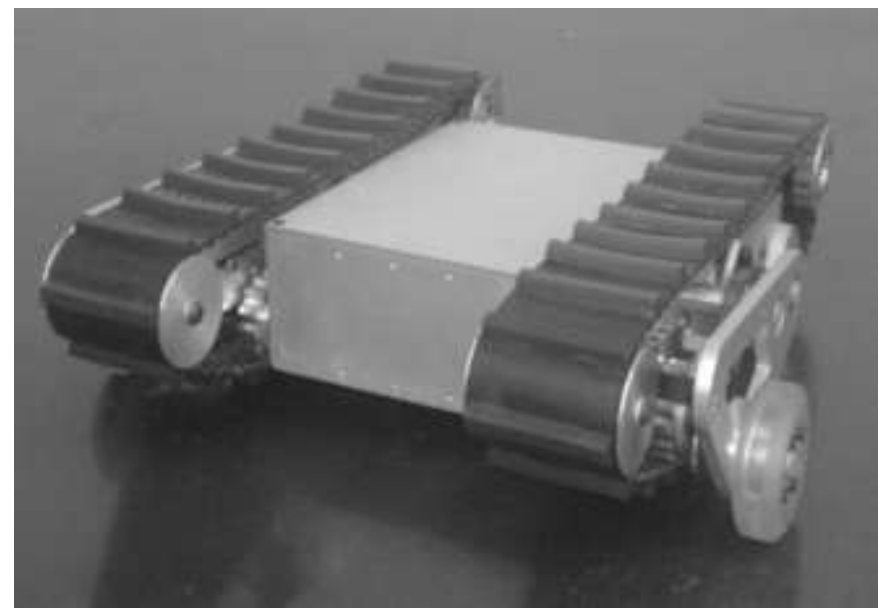

Fig 5: The NEZA-I robot
Terrain adaptive robot (see Fig.6) is a compound mobile robot with wheel and shoe. This robot has three motion modes, flat road driving mode. In the former mode, the track can be deformed according to the obstacle height. In the latter mode, the track deforms backward by 120 to ensure that the center of gravity of the robot can be moved backward to improve the motion stability. The maximum contour size of the robot is $1146 \mathrm{~mm} * 600 \mathrm{~mm} * 343 \mathrm{~mm}$. The maximum running speed is $7.3 \mathrm{~km} / \mathrm{h}$ in the wheel drive mode and $2.16 \mathrm{~km} / \mathrm{h}$ in the track drive mode. The net weight is $50 \mathrm{~kg}$ and the load is $20 \mathrm{~kg}$. Based on the principle of parallelogram, this type of robot track mechanism can reduce the number of driving motors and the total weight of the robot by using the passive adaptive method, and has better performance of obstacle avoidance.

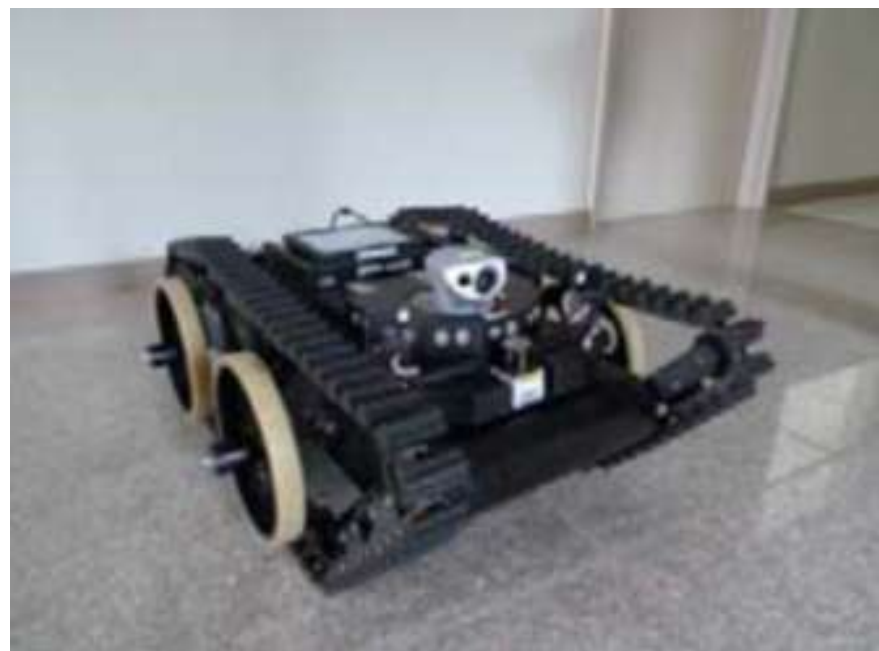

Fig 6: The terrain-adaptive robot

Deformation of what has been discussed above, it can be tracked mobile robot is walking mechanism in order to adapt to the unstructured environment and the new configuration, swing arm is often used to track, swinging rod, polygon mechanism to the deformation of the track and the fulfillment of wheel combined make its mobility and crosscountry performance is improved, but its structure is complex, more trouble control. The driving principle between fixed track and deformable track is similar, which will be further discussed in chapter 3 .

\section{STUDY ON THE DESIGN METHOD OF} WALKING MECHANISM CONFIGURATION

Based on the characteristics of the walking mechanism configuration of crawler mobile robots at home and abroad, various crawler robots are not unrelated to each other, but have certain relations and rules. Also mentioned the problem in section 2.2, such as single section double caterpillar has simple structure, can satisfy the requirement of general complex ground work, but its climb the slope, the gap of the barrier ability is poorer, the obstacle-surmounting performance, to improve its unilateral equipped with a pair of swing arm four crawler constitute the double festival, and 
it can climb high bigger obstacles, but its obstacle ability remains limited, again on the robot equipped with two swing arm to above constitute more section crawler, further enhance its capability of the obstacle negotiation. On the other hand, many deformable tracks are also derived from the above configurations. Therefore, various types of track walking mechanism configurations can be derived by extrapolation method, as shown in figure 7.

Fixed caterpillar type walking mechanism (a) adds swing arm to form two-section four-track mechanism (b) and three-section six-track mechanism (c); Triangular crawler (d) and trapezoidal crawler (e) are formed when the swing Angle of four and six crawler mechanisms is fixed. Replace the swinging arm of the triangular crawler with the selftensioning swinging arm of the crawler to form a deformable single-swinging arm crawler mechanism (f); If one side of the trapezoidal crawler mechanism is replaced by the crawler self-tensioned swinging arm, then the compound swinging arm crawler mechanism (g) is formed. The comprehensive configuration (f) and the configuration (g) are characterized respectively to form the doubleswinging arm mechanism (h). Therefore, the comparison method can be used to compare the characteristics of the derived crawler walking mechanism configuration to select or design the appropriate walking mechanism according to the needs of special tasks, as shown in table 1 .

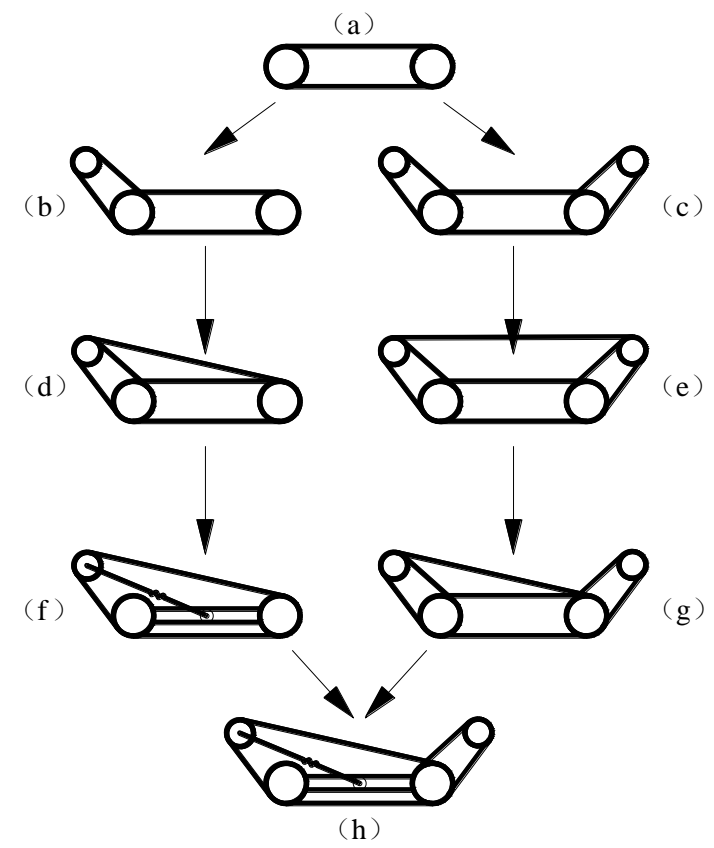

Fig 7: The extrapolated configuration

Table 1: The comparison among configurations

\begin{tabular}{|l|l|}
\hline $\begin{array}{l}\text { config } \\
\text { uration }\end{array}$ & Configurational characteristics \\
\hline a & $\begin{array}{l}\text { Need 1 power input, simple structure, poor } \\
\text { barrier performance. }\end{array}$ \\
\hline
\end{tabular}

\begin{tabular}{|l|l|}
\hline $\mathrm{b}$ & $\begin{array}{l}2 \text { power input is required, and the single } \\
\text { swing arm is introduced. Compared with the } \\
\text { configuration (a), it has a certain obstacle } \\
\text { clearance capability. }\end{array}$ \\
\hline $\mathrm{c}$ & $\begin{array}{l}3 \text { power input is required, and double } \\
\text { swinging arms are introduced. Compared } \\
\text { with configuration (b), the ability to } \\
\text { overcome obstacles is higher. }\end{array}$ \\
\hline $\mathrm{d}$ & $\begin{array}{l}1 \text { power input is required. Compared with } \\
\text { the configuration (c), the obstacle avoidance } \\
\text { performance is lower, but the track is in } \\
\text { contact with the ground, and the stability is } \\
\text { higher. }\end{array}$ \\
\hline $\mathrm{e}$ & $\begin{array}{l}\text { power input is required, which is a } \\
\text { trapezoidal structure. Compared with } \\
\text { configuration (d), it has similar performance. }\end{array}$ \\
\hline $\mathrm{f}$ & $\begin{array}{l}2 \text { power input is required. Compared with } \\
\text { the configuration (d), the caterpillar self- } \\
\text { tensioning swing arm mechanism is } \\
\text { introduced, and the performance of obstacle } \\
\text { crossing is higher. }\end{array}$ \\
\hline $\mathrm{g}$ & $\begin{array}{l}2 \text { power input is required. Compared with } \\
\text { configuration (e), one end is a swinging arm, } \\
\text { which has a higher performance. }\end{array}$ \\
\hline $\mathrm{h}$ & $\begin{array}{l}\text { 3 power input is required. Compared with } \\
\text { the configuration (g), the self-tensioning } \\
\text { swing arm of the crawler is increased to } \\
\text { make the crawler deformable. The contact } \\
\text { area between the crawler and the ground } \\
\text { increases and the stability is improved. } \\
\text { Compared with the configuration (f), a single } \\
\text { swing arm is added to improve the } \\
\text { performance. }\end{array}$ \\
\hline
\end{tabular}

\section{DISCUSSION AND RESEARCH PROSPECT}

When performing a variety of tasks, mobile robots need to adapt to the changeable and complex environment, which requires them not only to have a good walking mechanism to adapt to the changeable and complex unstructured environment, but also to have high mobility, motion stability and the ability to overcome obstacles. The unstructured environment is uncertain and higher requirements are put forward for the design of mobile robot configuration. The development trend is prospected and discussed:

(1) The research on crawler mobile robot is gradually mature, its walking mechanism is gradually diversified, and the research on the special robot technology applied in special environment has become a hot spot.

(2) To a specific environmental needs comprehensive characteristics of various types of mobile robots, without changing crawler robot under the premise of good crosscountry performance, adopts composite or shape-shifting robot composite structure, such as the wheel can be realized through the deformation of the track shoe type round flexible shoe pattern transformation in order to make up for the inadequacy of the crawler mobility, for the shoe leg type can be simplified by using a bionic legs or a simple and 
reliable institutions instead of biological leg in order to improve the obstacle-surmounting performance of crawler.

(3) The design of robot configuration shall also take into account the influence of energy consumption factors such as volume and quality. By optimizing the configuration of the mechanism and adopting light and reliable materials, the size and quality of the robot can be reduced to reduce energy consumption and extend its endurance and life.

(4) There are such problems as overturning, slippage and impact when the robot moves, and the research on its stability will also be the focus of the research on the structure design of the mobile robot.

\section{CONCLUSION}

Caterpillar mobile robot is of great significance in theoretical research and application. Especially in the context of the complex and volatile unstructured environment, a lot of work has been carried out at home and abroad. The current relevant research involves multiple disciplines and fields and is very active, so it is difficult to comprehensively review the work in various aspects. This paper tracked mobile robots in changeable moving characteristics in complex unstructured environment, mainly from the Angle of institutions tracked mobile robot at home and abroad research concerning the configuration design of traveling mechanism and progress were summarized and analysis, points out the existing problems of configuration design and internal laws, on this basis, from the perspective of personal future research direction and development trend were put forward.nd service life are improved, which greatly improves the efficiency of centrifugal pump and has certain reference value.

\section{REFERENCES}

[1] Song Yong-duan, Li Dan-yong, Cai Wen-chuan. "Mobile Robots and the Autonomic Technologies,"China Machine press., 2012.

[2] Si Yue-yuan. "Walking Mechanism Design and Kinematic Analysis of Wheel-Tracked Composite Robot," Machinery Design \& Manufacture.,vol.7, pp. 191-193, 2013.

[3] Cao Kai, etc. "Kinematics Analysis and Parametric Design of a Walking Mechanism of Bionic Biped Water-Walking Robot," Machinery Design \& Manufacture., vol.2, pp. 190-193, 2013.

[4] Ji Yang, Huo Guang-qing. "Research Status of Track-type Mobile Robots," Forestry Machinery \& Woodworking Equipment., vol. 10, pp. 7- 10, 2012.

[5] Dexter Duckworth. "Run the Robot Backward [C]// Proceedings of the IEEE International Symposium on Safety, Security, and Rescue Robotics (SSRR).," USA:IEEE., pp. 1-6, 2013.

[6] Tian Hai-bo. "Design and Implementation of Wheeltracked Mobile Robot," Modular Machine Tool \& Automatic Manufacturing Technique., vol. 7, pp. 1618, 2015.
[7] Sun Jiu-wei."Design Research of Multi-section Crawler-type Underground Coal Mine Search Robot," Coal Mine Machinery., vol. 30, pp. 20-23, 2009.

[8] Lv Guang-ming. "Design and motion analysis on variable parallelogram tracked mobile robot,"Shenyang: Shenyang Aerospace Universityl., 2013.

[9] Li Zhi-qing. "Development of a Transformable Wheel-track Robot with Self-adaptive Ability," Journal of Mechanical Engineering., vol. 47, pp. 110, 2011.

[10] Yoo-Seok Kim. "Autonomous Terrain Adaptation and User-friendly Tele-operation of Wheel-track Hybrid Mobile Robot," International Journal of Precision Engineering and Manufacturing., vol. 13, pp. 1781-1788, 2012. 